\title{
Análisis contrastivo del léxico del calzado en diccionarios especializados (español-francés-inglés): necesidades lexicográficas del traductor técnico desde una perspectiva multimodal
}

\author{
Contrastive Analysis of the Footwear Lexicon in Specialized Dictionaries \\ (Spanish-French-English): Lexicographic Needs of Technical Translator \\ from a Multimodal Point of View
}

Gisella Policastro Ponce [gisella.policastro@uco.es]

Universidad de Córdoba, España

\begin{abstract}
RESUMEN
Desde una perspectiva intermedia entre la lingüística y la terminográfica, el contenido especializado puesto a disposición de los usuarios a través de los diccionarios ha sufrido una significativa transformación, abarcando una nueva dimensión multimodal, en la que se evidencian de forma paralela nuevas exigencias por parte del traductor especializado. Con el presente estudio se pretende ofrecer una visión actualizada de las necesidades lexicográficas del traductor técnico desde un enfoque multimodal con vistas a la recurrencia a elementos no verbales que complementen el contenido verbal y satisfagan dichas demandas. Actualmente, los diccionarios especializados siguen presentando dificultades documentales e interpretativas para la actividad traductora, en la medida en que las particularidades de las que precisa el traductor difieren de las del público general y del lector especializado, a los que generalmente suelen ir destinados este tipo de herramientas de consulta. Basándonos en la propia praxis traductológica, nuestra premisa se tratará de evidenciar por medio de un análisis lexicográfico de dos términos especializados esenciales dentro del ámbito del calzado al objeto de manifestar las posibles dificultades que actualmente continúan presentando las herramientas lexicográficas para el traductor técnico y demostrar la necesidad de implementar recursos terminográficos multimodales.
\end{abstract}

\section{Palabras Clave}

Lexicografía; terminología; diccionarios especializados; multimodalidad; traducción profesional

\begin{abstract}
From an intermediate perspective between linguistics and terminology, the specialized content which is made available to users through dictionaries has undergone an important transformation embracing a new multimodal dimension, in which new specialised translations needs run in parallel. This study aims to provide an updated view of the lexicographical demands of the technical translator from a multimodal approach, by means of implementing non-verbal elements that complement verbal content and satisfy these demands. Nowadays, specialized dictionaries still remain insufficient in terms of documentation and interpretation
\end{abstract}


needs for the translation activity, insofar as the particularities required by the translator differ from the needs of the general public and the specialized reader, to whom this type of consultation tool is generally addressed. Based on our own translating experience, our premise will be demonstrated by means of a lexicographical analysis of two essential specialized terms within footwear field in order to highlight the potential difficulties that lexicographical tools currently continue to present for technical translators, and the importance of using multimodal terminological resources in this regard.

\section{KEYWORDS}

Lexicography; terminology; specialized dictionaries; multimodality; professional translation

RECIBIDO 2020-05-22; ACEPTADO 2020-10-01

\section{Introducción}

La transmisión del contenido especializado basada únicamente en el lenguaje, como tradicionalmente se concebía el concepto comunicativo, comenzó a resultar poco convincente hace algunas décadas, concretamente a partir de los setenta, con el surgimiento de la Teoría Multimodal. La realidad discusiva que plantea esta teoría se aleja de la tendencia monomodal centrada exclusivamente en el lenguaje y renueva el concepto comunicativo del texto al concebirlo en contacto con y «dentro de un dominio sociocultural dado» (Kress y van Leeuwen 2001: 1), de tal forma que crea significado a partir de los diversos recursos semióticos que tiene a su alcance.

La renovación que ofrece la implementación de la multimodalidad en los diferentes escenarios comunicativos se ha puesto de manifiesto mediante la fusión de diversos modos de expresión, verbal y no verbal, que se materializan en un único formato textual. Desde una perspectiva lexicográfica, el contenido especializado puesto a disposición de los usuarios a través de los diccionarios también ha sufrido una transformación, abarcando una nueva dimensión multimodal en la que se evidencian de forma paralela nuevas necesidades por parte del traductor especializado (Cabré, Feliu y Tebé 2001; Prieto Velasco y Faber 2012).

Conscientes de la creciente relevancia de los formatos multimodales como herramienta de comunicación satisfactoria y adaptada a las necesidades actuales de los usuarios, el presente trabajo tiene la intención de retomar el asunto del cual la literatura científica se ha ocupado en varias ocasiones: los límites de los repertorios lexicográficos en el marco de la traducción especializada. En esencia, nuestra investigación se centra en ofrecer una visión real de las necesidades lexicográficas del traductor técnico, centrando nuestra atención en la presencia de elementos multimodales y el modo en el que estos pueden contribuir a una mejora interpretativa de la definición del término en cuestión.

Teniendo en cuenta las consideraciones que expone Sánchez Cárdenas (2008, s. p.), resulta evidente que los diccionarios especializados continúan interponiendo obstáculos a la labor traductora: 
A pesar del significativo desarrollo de herramientas destinadas a la traducción de nuevas formas de texto -y por lo tanto nuevas formas de traducción- [...], la investigación en lexicografía bilingüe no han dado todavía el salto hacia estas nuevas formas de producción textual de una manera satisfactoria. Parece ser que la visibilidad de los resultados de la investigación lingüística no van actualmente a la par de aquellos surgidos de la innovación tecnológica.

En este estudio, que parte de la propia experiencia práctica, hemos seleccionado dos artículos lexicográficos que corresponden a dos términos especializados propios del sector del calzado ( pala y caña) y que constituirán la base de nuestro corpus, extraídos a partir de cuatro diccionarios especializados en formato bilingüe.

Partiremos de la reflexión sobre la eficacia e idoneidad de dichas herramientas lexicográficas en relación con las exigencias concretas del traductor mediante la consulta de dichos términos, acompañada de un análisis lexicográfico contrastivo en español-francés-inglés. Dicho estudio revelará su carácter restrictivo, que se limita a la propuesta de equivalentes y/o descripciones semánticas y «no contempla(n) la diversidad de objetivos del producto terminográfico» (Gómez González-Jover y Vargas Sierra 2004, s. p.). De esta manera, trataremos de identificar cuáles han sido las dificultades encontradas y determinar de forma concreta las necesidades traductológicas en materia lexicográfica. Todo ello con el propósito de poner de manifiesto la conveniencia de utilizar recursos terminográficos multimodales en las obras lexicográficas en favor de la actividad traductora.

Como fin último, el presente estudio sugiere la posibilidad de ampliar dicha investigación, profundizando en la aplicación de la teoría multimodal al diseño de diccionarios de traducción especializados en el ámbito técnico, inspirada en las investigaciones de autores como Cabré, Feliu y Tebé (2001), Lew (2010), o Tarp (2014), que han demostrado la importancia de los elementos no lingüísticos en la lexicografía especializada.

\section{El traductor técnico y recursos de consulta: diccionarios de traducción especializados}

Todo traductor técnico, algo que se cumple también para cualquier traductor especializado, debe asumir las mismas competencias cognitivas, lingüísticas y expresivas que las de sus homólogos especialistas en cada una de las ramas del saber implicadas en el texto original (en el caso práctico que nos ocupa nos referiríamos al especialista en el sector del calzado) para garantizar la correcta inteligibilidad del texto meta y la adecuada transmisión del mensaje por parte del lector. El uso de materiales documentales permite, al traductor, alcanzar dicho nivel de domino del área del saber que se trate, al tiempo que le ayudan a resolver las posibles complicaciones terminológicas, lingüísticas, gramaticales, contextuales, conceptuales o convenciones textuales presentes en el texto susceptible de ser traducido (Gamero Pérez 2001).

En el marco de nuestro estudio, abordamos las fuentes lingüísticas, (siguiendo la clasificación de Gonzalo García 2004), sucintamente, los diccionarios bilingües, la obra de consulta filológica por antonomasia que cuenta con el mayor repertorio de recursos lingüísticos y a la que se suele acudir con mayor frecuencia durante el proceso de traducción para la resolución de cuestiones 
de tipo terminológico. Resulta axiomática la ineludible recurrencia a este tipo de herramientas por parte del traductor técnico, como consecuencia de elevada concentración de terminología especializada (Cabré 2010) en los textos con los que trabaja. Es un componente particularmente destacable de los textos técnicos la precisión terminológica con valor unívoco, con la que se pretende eludir cualquier tipo de ambigüedad, así como la abundancia de los elementos nominales (García Ubago 2012).

Dicha evidencia queda de manifiesto en la reflexión de González-Jover y Vargas Sierra (2004, párr. 16), que se cita a continuación:

La comprensión de textos está íntimamente ligada con la competencia cognitiva [...], y supone un conocimiento de las nociones que articulan el conocimiento propio de la disciplina de especialidad y de las relaciones conceptuales que se producen en la misma. El traductor debe poder tener acceso a este tipo de información, aunque con un nivel de especialización que difiere del especialista, por lo que el diccionario de especialidad ha de concebirse como un instrumento de apoyo en este sentido. La definición, que sirve para describir el concepto que representa el significado de una denominación, así como el área temática, que permite discriminar entre diferentes significados de una unidad terminológica potencialmente polisémica, y asociarla con el equivalente más adecuado en la lengua meta (Cabré 2002: 170), o las relaciones entre conceptos, son algunas de las informaciones relacionadas con el universo conceptual del ámbito especializado que sirven al traductor en la comprensión del texto. Sin este conocimiento, el texto traducido difícilmente puede alcanzar unos niveles adecuados de calidad.

Esta contribución anteriormente citada se presenta particularmente interesante dado que trata importantes cuestiones a considerar en nuestro trabajo de investigación, gracias a la proximidad del planteamiento ${ }^{1}$, al mismo tiempo que arroja luz sobre el enfoque terminográfico del presente estudio. Vale la pena señalar en la cita anterior que la descripción sistemática que ofrecen los diccionarios en su organización conceptual tradicional difiere de las exigencias específicas del traductor que, amén de encontrar en este tipo de fuentes una equivalencia a las unidades léxicas especializadas en sus lenguas de trabajo, exigen un modo de representación que no se limite a la correspondencia conceptual, sino que complemente su comprensión acerca del mismo, interrelacione las unidades léxicas entre sí, según los parámetros lingüísticos y extralingüísticos pertinentes y permita la inclusión de diferentes tipos de representación no verbal. Todo ello en aras de una mejora comprensiva e interpretativa del contenido léxico por parte del usuario traductor.

Abaitua (2001, párr. 16) plantea también la cuestión de la exhaustividad en los diccionarios como «un problema antiguo e insoluble», en referencia a la concepción de la lengua como un elemento vivo, frente a la concepción de los diccionarios como «depósitos estáticos», otro aspecto que de igual modo afecta negativamente a la labor documental del traductor, que persigue

1 La cita de las autoras González-Jover y Vargas Sierra (2004) se enmarca dentro de una contribución asociada a un proyecto de investigación de la Universidad de Alicante titulado Creación de una base de datos terminológica de algunos sectores industriales de la Comunidad Valenciana, concedido por el Ministerio de Educación, cuyo director era Enrique Alcaraz Varó. En esta publicación se plantea una propuesta metodológica para la elaboración de diccionarios de índole técnica, en concreto una de las obras generadas es el Diccionario de Términos del Calzado e Industrias Afines, lo que posiciona a dicho estudio muy próximo a nuestro trabajo de investigación. Cabe añadir que dicha obra de consulta lexicográfica forma parte del objeto de estudio del presente trabajo. 
la consulta de información actualizada en la materia y contribuye a la correcta traslación de los conocimientos especializados de una lengua a otra.

Comenzábamos el presente apartado aludiendo a la equiparación, a nivel cognitivo, lingüístico y expresivo, del traductor al del especialista, ambos dentro de un mismo campo de saber; si bien el grado de accesibilidad de cada uno al tipo de fuente de consulta citada no es comparable. Si asumimos dicha consideración, resultaría cuanto menos plausible facilitar una representación de la información especializada en formato bilingüe materializada a través de diferentes niveles de significación y adaptada a las demandas específicas de cada usuario que habitualmente haga uso de los diccionarios especializados como herramienta de consulta, puesto que resulta inaccesible satisfacer las necesidades de cada uno por igual.

Lejos de posicionar al léxico especializado en un segundo plano, nuestra reflexión trasciende el plano lingüístico, tratando de ofrecer a los elementos de significación verbal un complemento que contribuya de una forma más completa y comprensiva a su interpretación, comprensión y traducción.

\section{Análisis contrastivo del léxico del calzado en diccionarios especializados (español-francés-inglés)}

No son pocas las veces en las que los traductores técnicos se enfrentan a numerosas complicaciones a la hora de identificar el significado de un término específico o encontrar un equivalente unívoco en la lengua de destino dentro de un contexto dado (Sánchez Cárdenas 2008) cuando recurren a fuentes de consulta lexicográfica.

En el presente trabajo trataremos de identificar cuáles son los posibles problemas que el traductor técnico puede encontrar hoy en día durante el proceso de documentación terminológica cuando hace uso de diccionarios bilingües ${ }^{2}$ como instrumento indispensable para satisfacer sus necesidades lexicográficas. En este sentido, al objeto de ilustrar dichas dificultades, hemos considerado oportuno tomar como punto de partida un caso extraído de la propia praxis traductológica: la consulta, tanto en francés como en inglés, de dos términos de uso común dentro del sector del calzado, pala ${ }^{3}$ caña $a^{4}$ que presentaron inconvenientes en términos lexicográficos durante el ejercicio práctico de la traducción de especificaciones técnicas de este tipo de productos. Por lo tanto, a modo de aclaración, exponemos a continuación la metodología que se utilizó en el citado proyecto de traducción, que evidentemente es la misma sistemática que utilizamos para el presente estudio, en tanto en cuanto nuestra investigación se fundamenta en dicho caso práctico.

Durante el proceso documental previo a la tarea traductora se experimentó cierta confusión al consultar las herramientas al servicio de los profesionales de la lengua ${ }^{5}$. En primer lugar, se realizó

2 Cabe matizar que se ha limitado el campo de estudio a los diccionarios bilingües por cuestiones meramente limitativas del espacio disponible.

3 De acuerdo con el Diccionario de la lengua española, en el caso que nos ocupa, el término «pala» hace referencia a: 6. f. Parte superior del calzado, que abraza el pie por encima.

4 De acuerdo con el Diccionario de la lengua española, en el caso que nos ocupa, el término «pala» hace referencia a: 6 . f. Parte de la bota o de la media que cubre entre la rodilla y el pie.

5 Otra cuestión que también generó ciertas dificultades fue la polisemia que caracteriza a ambos conceptos, en 
una selección de recursos lexicográficos tanto monolingües como bilingües, haciendo uso tanto de diccionarios especializados como no especializados, tomando como referencia las siguientes consideraciones:

- La temática debía enmarcarse en la medida de lo posible en el sector del calzado. Se convino además que, en el caso de no encontrar fuentes documentales propias de esta área de conocimiento, se podría ampliar el rango a recursos relacionados con el sector textil.

- Partiendo de nuestra premisa, se trató de seleccionar fuentes que contaran con información gráfica, en la medida de lo posible.

- La combinación lingüística debía incluir los siguientes idiomas: español, inglés y francés, con independencia de su combinación.

A partir de dicha búsqueda, que resultó más ardua de lo previsto debido a la escasa disponibilidad de diccionarios que cumplieran con los requisitos antemencionados, se seleccionaron un total de cuatro fuentes documentales lexicográficas relativas al calzado que se encontraban disponibles en formato bilingüe:

- Las dos versiones del Diccionario de términos del calzado inglés-español/español-inglés y francés-español/español-francés, elaborado por la Cámara de Comercio de Alicante, que se encuentra disponible en formato bilingüe francés-español, por un lado, e inglés-español, por otro, existiendo por tanto dos versiones y que cuenta con la presencia de información gráfica.

- Le grand dictionnaire terminologique, que ya en su propia presentación se define como «une banque de fiches terminologiques» (un banco de fichas terminológicas) elaborado por la Office québécois de la langue française (Oficina quebequesa de la lengua francesa). Cada registro proporciona información sobre un concepto relacionado con un campo especializado y presenta el término en francés, inglés y, en ocasiones, en otros idiomas. Asimismo, algunas entradas incluyen ilustraciones.

- El Diccionario de términos del calzado e industrias afines, editado por Alcaraz et al., como resultado del proyecto de investigación de la Universidad de Alicante, titulado «Creación de una base de datos terminológica de algunos sectores industriales de la Comunidad Valenciana, y disponible exclusivamente en formato bilingüe inglés-español». Uno de los aspectos a destacar de este recurso es la ausencia de elementos multimodales que aporten significado no verbal a las unidades de significación verbal que conforman el diccionario. Precisamente se plantea como interesante este aspecto al objeto de poder compararlo con las demás opciones $y$, de este modo, valorar la idoneidad de nuestra premisa.

Una vez planteada la metodología e identificadas las herramientas de consulta, procedemos a analizar los resultados siguiendo el planteamiento organizativo que se detalla a continuación:

función de su uso como palabra dentro del lenguaje general o como término dentro del lenguaje especializado. Un aspecto que se ha tomado en consideración y se reservará a futuras investigaciones. 
1. En primer lugar, se mostrarán los hallazgos obtenidos para cada entrada durante el proceso de consulta de los términos pala y caña, respectivamente, en cada una de las fuentes documentales previamente citadas.

2. A la luz de lo anterior, se expondrán y comentarán de forma detallada las dificultades que las fuentes lexicográficas consultadas han presentado para el traductor. Se hará especial hincapié en demostrar que el uso de recursos multimodales en obras lexicográficas contribuye de forma significativa a la actividad traductora.

3. Por último, se recogerán en una tabla, a modo de resumen, las principales insuficiencias lexicográficas encontradas en cada diccionario consultado.

\subsection{Resultados de la consulta de los términos pala y caña}

La identificación del equivalente terminológico de los términos pala y caña en las lenguas de destino, inglés y francés ofreció los siguientes resultados en cada uno de los diccionarios bilingües consultados, tal y como se muestra a continuación:

\section{Entradas para el término pala}

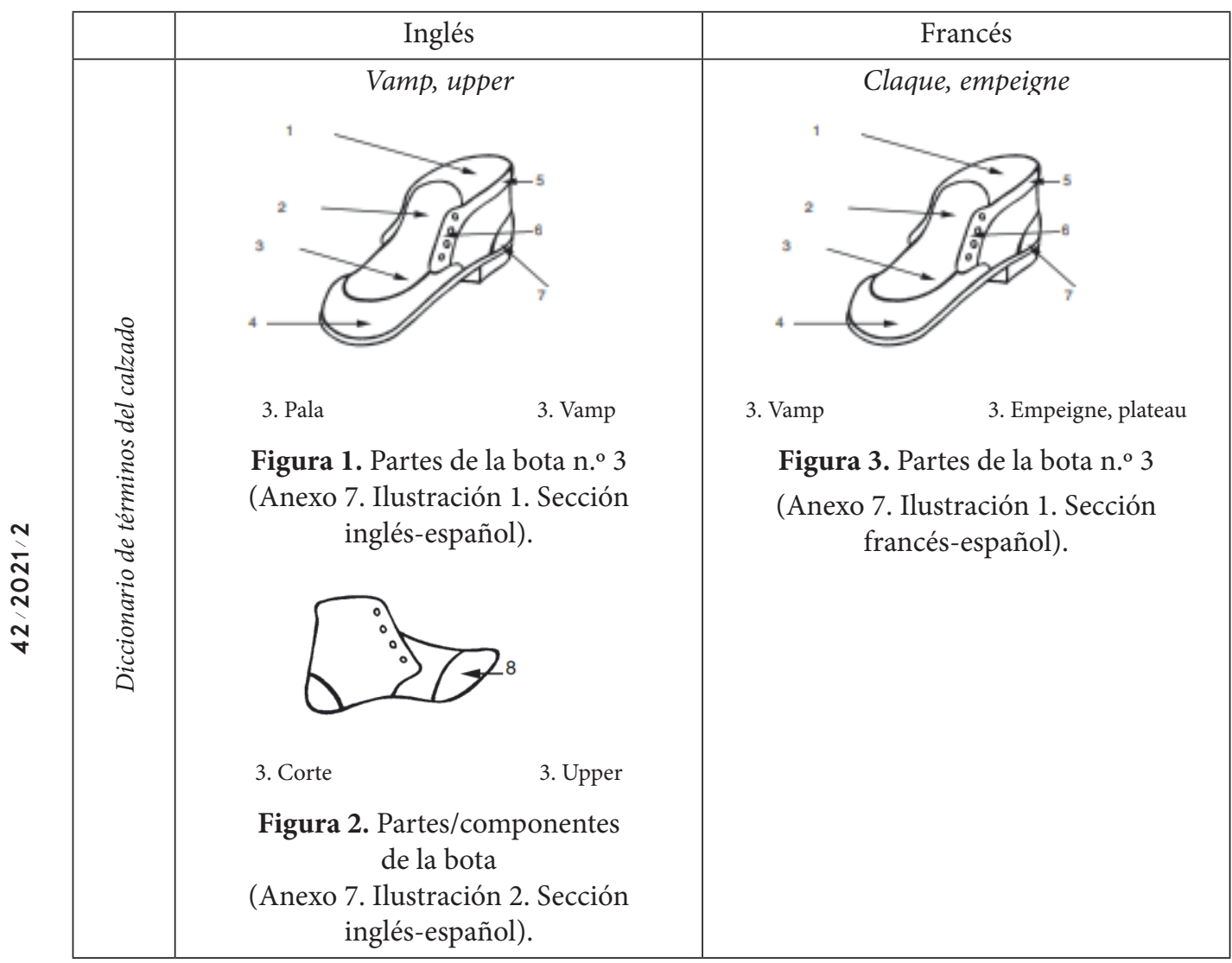




\begin{tabular}{|c|c|c|}
\hline & Inglés & Francés \\
\hline \multirow{4}{*}{ 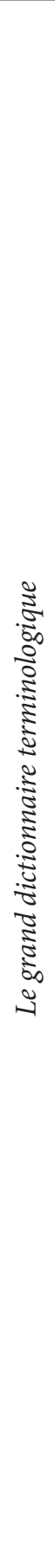 } & Vamp & $\begin{array}{c}\text { Claque } \\
\text { Se identifican varias opciones dentro } \\
\text { del propio diccionario que se enumeran } \\
\text { a continuación según la fuente y por } \\
\text { orden cronológico: }\end{array}$ \\
\hline & Foxing & $\begin{array}{c}\text { Opción 1 } \\
\text { Fuente: } \\
\text { Association française } \\
\text { de normalisation, } 1977 \\
\text { Definición: } \\
\text { Bande de renforcement destinée à protéger } \\
\text { le bord inférieur de la tige des éraflures, } \\
\text { placée le long du bord inférieur de celle- } \\
\text { ci avant, pendant ou après la pose de la } \\
\text { semelle. } \\
\text { Se sugieren los términos claque y bande. }\end{array}$ \\
\hline & \multirow[t]{2}{*}{ Vamp } & $\begin{array}{c}\text { Opción 2 } \\
\text { Fuente: } \\
\text { Office québécois } \\
\text { de la langue française, } 1982 \\
\text { Definición: } \\
\text { Partie principale de lavant-pied d'une } \\
\text { chaussure à laquelle se fixe éventuellement } \\
\text { une languette ou un système de fermeture. } \\
\text { Se sugieren los términos } \\
\text { claque y empeigne. } \\
\text { Ilustración: }\end{array}$ \\
\hline & & $\begin{array}{l}\text { Figura 4. Parte del calzado denominada } \\
\text { claque en francés. }\end{array}$ \\
\hline
\end{tabular}




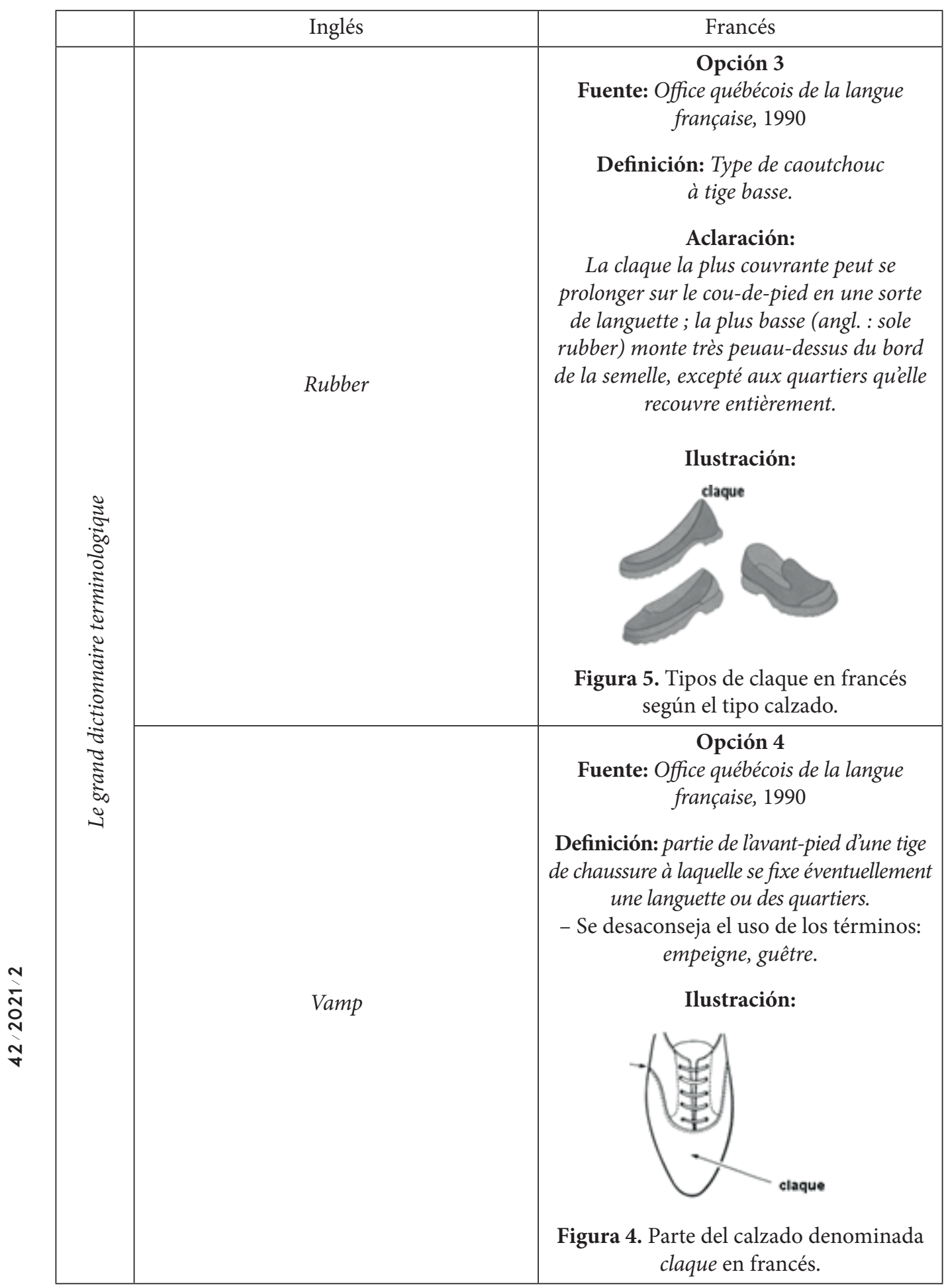




\begin{tabular}{|c|c|c|}
\hline & Inglés & Francés \\
\hline 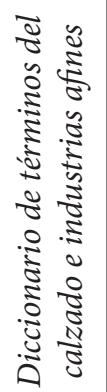 & Vamp,Upper & No disponible \\
\hline
\end{tabular}

Tabla 1. Resultados comparativos obtenidos tras la consulta del término pala en los diccionarios que conforman el corpus objeto de estudio.

岂 


\section{Entradas para el término caña}

\begin{tabular}{|c|c|c|}
\hline & Inglés & Francés \\
\hline 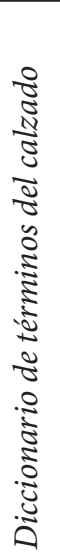 & $\begin{array}{l}\text { Se identifican tres voces: } \\
\text { - caña (bota): leg, legging, } \\
\text { quartertopline } \\
\text { - caña (zapato): quarter } \\
\text { - caña de la bota: top of bootleg }\end{array}$ & $\begin{array}{l}\text { 5. Quartier } \\
\text { Figura 6. Partes de la bota n. } 5 \\
\text { (Anexo 7. Ilustración 1. Sección } \\
\text { francés-español }\end{array}$ \\
\hline & & $\begin{array}{c}\text { Quartier } \\
\text { Aparecen varios resultados, que } \\
\text { se clasificarán según Fuente y por } \\
\text { orden cronológico, dentro del propio } \\
\text { diccionario: }\end{array}$ \\
\hline 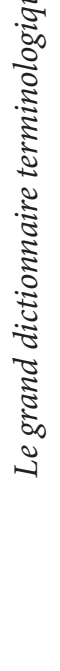 & Quarters & $\begin{array}{c}\text { Opción 1 } \\
\text { Fuente: Association française de } \\
\text { normalisation, } 1977 \\
\text { Definición: Partie arrière de la tige, qui } \\
\text { peut (peuvent) comporter un ou deux } \\
\text { éléments. } \\
\text { Aclaración: } \\
\text { Ce terme a pour origine l'emploi de } \\
\text { quatre éléments dans la fabrication des } \\
\text { parties arrière d'une paire de bottes ou de } \\
\text { chaussures. } \\
\text { Ilustración: }\end{array}$ \\
\hline
\end{tabular}




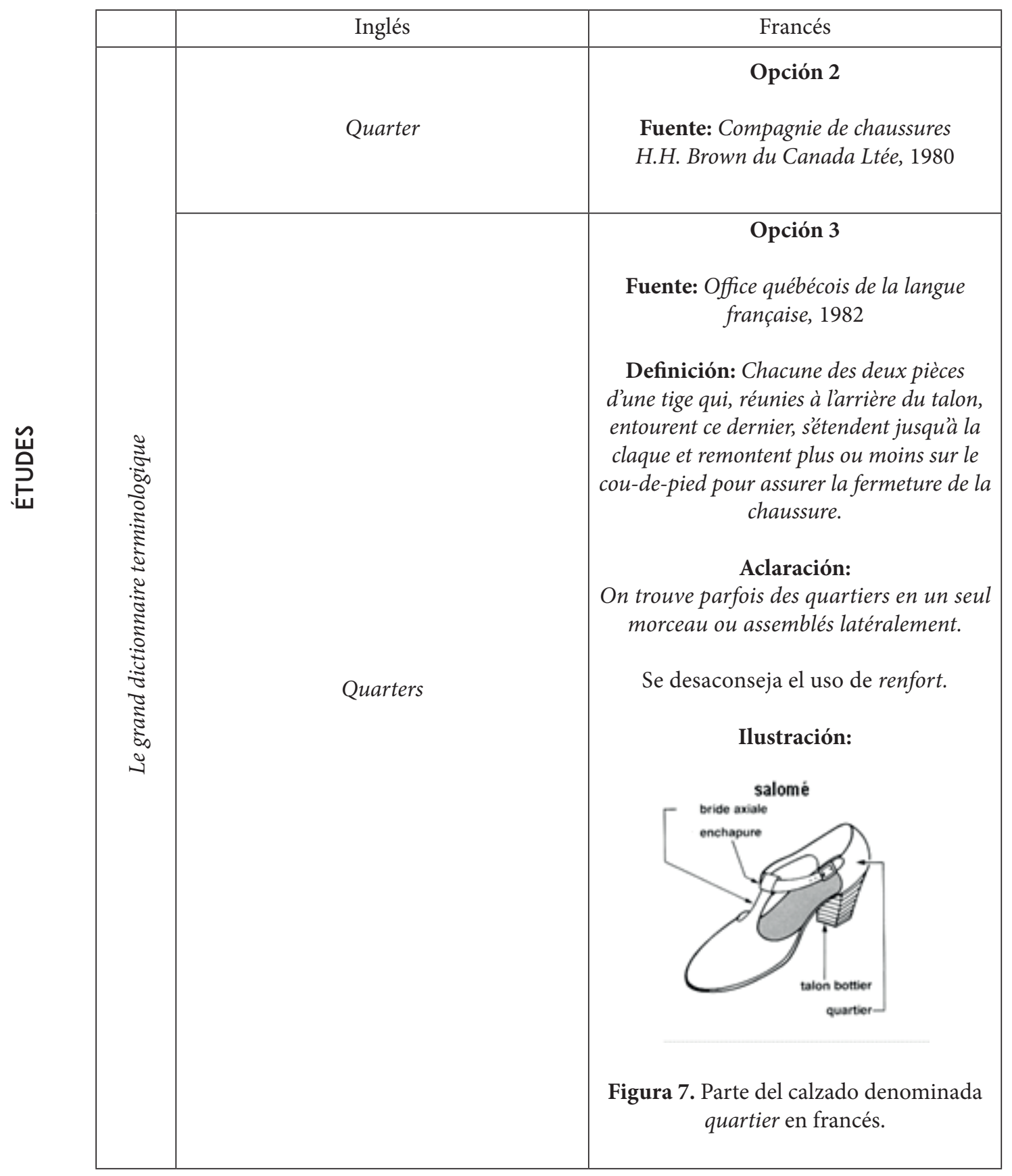




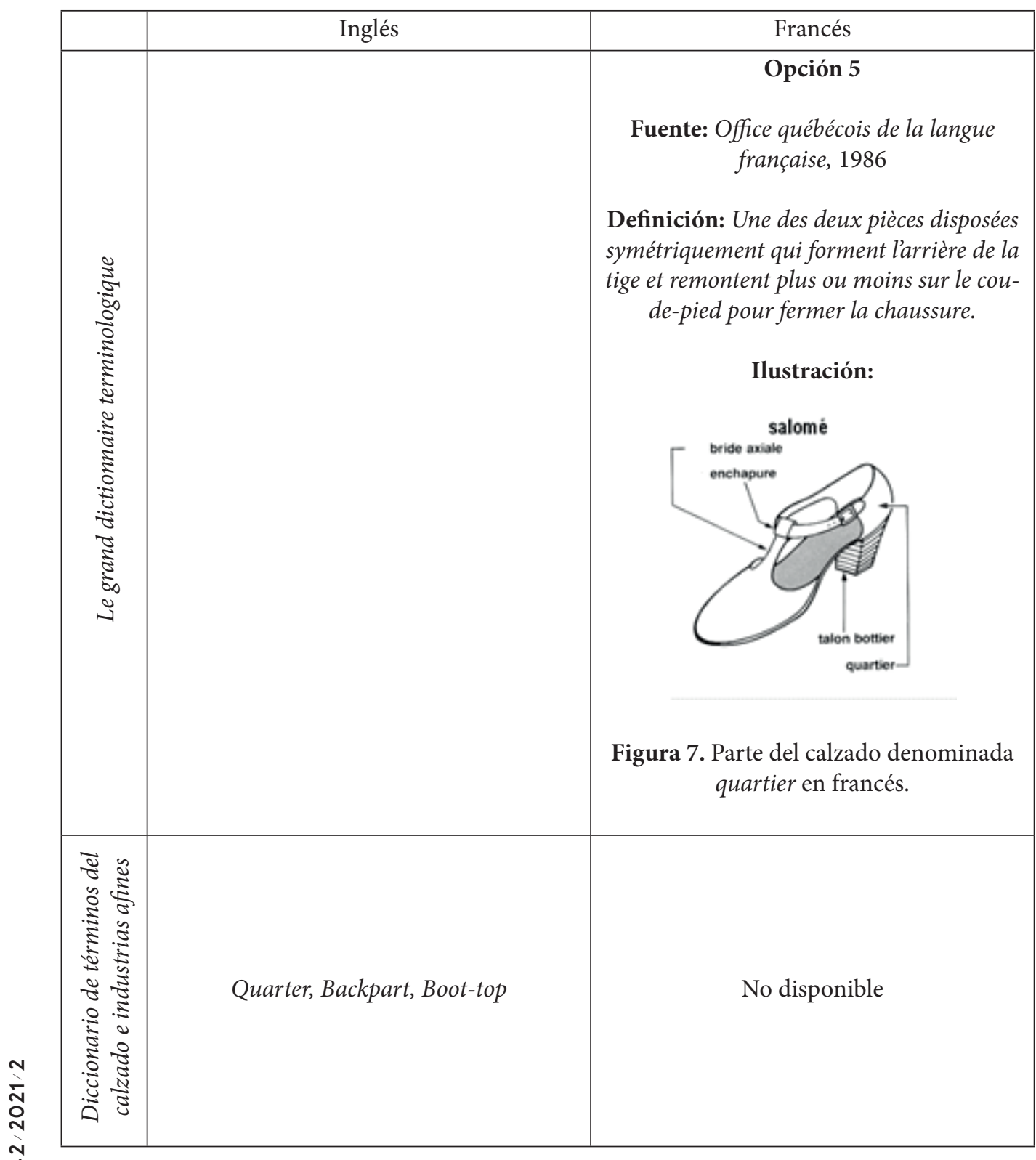

Tabla 2. Resultados obtenidos tras la consulta del término caña en los diccionarios que conforman el corpus objeto de estudio. 


\subsection{Dificultades encontradas en las fuentes lexicográficas consultadas}

En esta segunda fase nos centraremos en comentar en detalle los resultados de la consulta efectuada durante el proceso de documentación necesario para acometer el proyecto de traducción antemencionado, con miras a valorar si es posible determinar una opción terminológica que nos resulte convincente y satisfactoria. Esto nos permitirá valorar si dichas herramientas lexicográficas resultan eficaces ${ }^{6}$ para que el traductor pueda interpretar correctamente el concepto y encontrar un equivalente terminológico en cada idioma. Asimismo, identificaremos cuáles son los inconvenientes que estas fuentes pueden suponer para el traductor técnico.

a. En lo que respecta al término pala, en inglés, nos encontramos con los términos vamp, upper, foxing y rubber; y en francés, claque, empeigne y plateau.

i. Comenzamos por el Diccionario de términos del calzado, en concreto, por la versión inglesa. La Tabla 1 muestra que el único equivalente en inglés que aparece en la entrada del término pala es vamp. Sin embargo, consideramos pertinente efectuar una búsqueda general de las entradas que pudieran contener el término pala y se apreció la existencia de otras voces que en su equivalente en inglés no utilizaban vamp sino upper para hacer referencia, aparentemente, al mismo concepto, además sin ningún tipo de comentario explicativo (p. ej.: pala recortada: cutupper; compensar estirado de la pala: to anticipate stretching of upper; reforzar las palas: to back the uppers; puntear la pala: to prickuppers, entre otros casos). Por consiguiente, se estima conveniente proceder a la búsqueda del término upper en esta herramienta, lo que revela los siguientes resultados:

- upper: corte (aparado);

- upper: corte; parte superior; pala.

Igualmente, al buscar el término vamp, encontramos:

- vamp: pala

- $\operatorname{vamp}($ round): corte, pala

También se encontró el término broguewing asociado a pala y corte tope.

En lo que respecta a la presencia de elementos semióticos, en el Anexo n. ${ }^{\circ} 7$ del citado diccionario figuran varias ilustraciones en las que se indican las partes del calzado, a las que se les ha asignado un número, junto con sus propuestas de traducción en inglés. En el número 3 de la Figura 2 se identifica con el término vamp, en inglés, y pala en español, mientras que en la Figura 2, el número 8 se identifica en inglés con upper y en español con corte.

Como podemos comprobar, a pesar de las inconsistencias encontradas en las entradas del diccionario, resultó ser la información gráfica la que aparentemente esclareció algo más la identifica-

6 Consideramos oportuno aclarar que dicho análisis se lleva a cabo desde un punto de vista traductológico y multimodal, sin entrar a considerar su idoneidad como herramienta de consulta para otro tipo de usuario y, por supuesto, desde el más absoluto respeto y reconocimiento a la contribución lexicográfica de dichas obras. 
ción del término. La identificación visual del término nos permitió reconocer con precisión a qué parte del calzado hace referencia cada concepto.

Por otra parte, la versión en lengua francesa ofrece soluciones diferentes. Para empezar, tal y como se indica en la Tabla 1, se han encontrado hasta un total de tres propuestas terminológicas diversas (claque, empeigne y plateau) que, en un principio, parecen tener la misma significación. Al buscar el término pala en esta fuente, se ofrecen dos propuestas: claque y empeigne. Sin embargo, al realizar la búsqueda de francés a español de ambos términos, observamos que para claque solo se ofrece pala como equivalente en español, mientras que para empeigne aparece también empeine.

En la versión francesa también contamos con la presencia de elementos multimodales. En el mismo Anexo n. ${ }^{\circ} 7$ que corresponde igualmente a una serie de ilustraciones acompañadas por un número que identifica las diferentes partes del calzado, en francés y español, resulta curioso destacar que, como se observa en la Figura 2, el número 3 que corresponde a la pala, aparece en francés traducido solo como empeigne, plateau, sin mencionar el término claque. Por su parte, el término plateau, que no aparecía como equivalente de pala en francés, sí aparece en la ilustración. Ello nos lleva a buscar este término en otras entradas del diccionario y detectamos que aparece también en los siguientes casos: plateau de mocassin, traducido al español como plastrón de mocasín y forme à plateau, traducido al español como horma de mocasín. En ninguna otra entrada del diccionario aparece como equivalente de pala, salvo en la ilustración arriba mencionada.

Por último, dado que en la versión inglesa aparecía el término corte, se procedió también a indagar sobre la presencia de dicho término en la versión francesa del mismo diccionario, para comprobar si aparecía en alguna entrada y, en caso afirmativo, qué traducción tenía en francés. Encontramos el término corte traducido en francés como tige. Por consiguiente, buscamos en la sección francés-español y solo encontramos el siguiente resultado: tige façon mocassin: pala mocasín).

A modo de valoración general, estimamos que estos resultados generan cierta confusión a la hora de poder determinar un equivalente unívoco. Los casos de incoherencia terminológica se detectan tanto desde el español al francés o al inglés como del francés o el inglés hacia el español. Dicha discordancia se refleja en las ilustraciones, influyendo negativamente en la cohesión terminológica. No obstante, la identificación visual del término contribuye a una mejora interpretativa, ejerciendo su funcionalidad de forma correcta.

Asimismo, podríamos añadir que la ausencia de definiciones dificulta la comprensión del contenido terminológico, que podría verse complementado por los elementos multimodales correctamente cohesionados internamente con las entradas del propio diccionario.

ii. Le grand dictionnaire terminologique utiliza como primera lengua el francés, ofreciendo las definiciones de los conceptos en este mismo idioma, junto con la equivalencia terminológica en otras lenguas (inglés, en la mayoría de los casos, junto con español, catalán, gallego, italiano, portugués, rumano y latín). Es posible igualmente seleccionar como parámetro de búsqueda un dominio específico.

Se realizó la búsqueda del término pala en español, pero no resultó satisfactoria, puesto que este recurso no contemplaba este vocablo dentro del dominio del calzado. De manera que se optó por buscar las equivalencias terminológicas proporcionadas por el Diccionario de términos del calzado, en inglés (vamp, upper) y en francés (claque, empeigne y plateau). 
En primer lugar, se realizó la búsqueda del término vamp que, como se observa en la Tabla 1, proporcionó varios resultados (opción 1: foxing, opción 2: vamp, opción 3: rubber; opción 4: vamp) clasificados según la fuente y año de publicación de esta, acompañado en cada caso por una definición, y que procedemos a revisar a continuación.

Tomando como referencia las definiciones aportadas, la opción 2 y 4 (vamp) son las que cumplen con el significado del término en contexto. En ambas el término equivalente en francés es claque.

En nuestra exploración se detectó que, al igual que en el Diccionario de términos del calzado (versión inglesa), el término vamp aparece también como equivalente del término francés empeigne, que a su vez va acompañado de la siguiente definición: "partie avant de la tige située en avant du cou-de-pied». La definición contiene términos especializados desconocidos (tige y coude-pied) que dificultan la comprensión del significado del término inicial, un inconveniente que podría resolverse si dicha enunciación estuviera acompañada de una ilustración que permitiera identificar a qué parte del calzado hace referencia. Ello obliga a realizar una nueva búsqueda de estos conceptos, con la posibilidad de volver a encontrarnos con nuevos términos desconocidos. Por cuestiones de espacio, no se considera oportuno incluir en el presente trabajo los resultados obtenidos tras la consulta de dichos términos.

Descartamos la opción 1, en la que se propone el término foxing, porque la definición no concuerda con las demás ni con el significado de pala. No obstante, para reforzar dicha postura, se buscó foxing en este diccionario, dentro del dominio del calzado, y se encontraron los resultados siguientes ${ }^{7}$ :

- foxing EN • talonnette FR

Fuente: Compagnie de chaussures H.H. Brown du Canada Ltée, 1980

Sin definición y sin ilustración

- outside counter EN • talonnette de dessus FR

Fuente: Renaud, Micheline, 1985

Definición: «Partie arrière des quartiers constitués d’une ou deux pièces rapportée sousimulées entourant le talon».

En este caso en concreto, si analizamos las ilustraciones que se aportan, (tanto en inglés (Figura 8) como en francés (Figura 9)), ninguna de ellas coincide con la parte de la pala. Sin embargo, se observa que la parte de la pala, en ambas imágenes, ha sido identificada en inglés como vamp y en francés como claque.

7 Dichas entradas solo incluían como equivalente los términos en francés. 


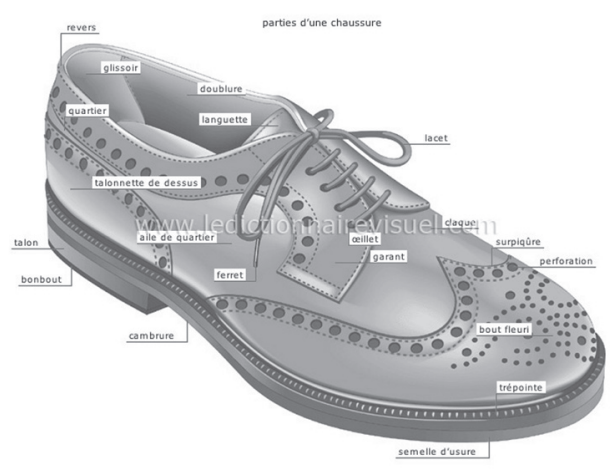

Figura 8: Partes del calzado en francés.

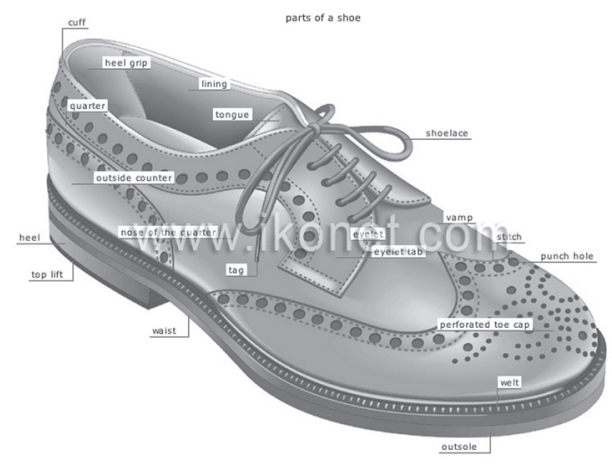

Figura 9: Partes del calzado en inglés.

Nuevamente nos encontramos con el caso de que, a pesar de que exista una incongruencia terminológica, gracias a la ilustración ha sido posible descartar una de las equivalencias propuestas y comenzar a considerar la posibilidad de haber encontrado el equivalente terminológico del concepto que estábamos buscando, en la medida en que los resultados del diccionario anterior apuntaban a la misma dirección.

La opción 3, en la que se propone el término rubber, se descarta igualmente por no concordar la definición en francés con el concepto de pala. De igual modo, para corroborar nuestra decisión, se procedió a la búsqueda del término en inglés en este mismo diccionario y se encontraron otras dos propuestas igualmente alejadas del término pala:

- overshoe EN • couvre-chaussure FR

Fuente: Office québécois de la langue française, 1982

Definición: «Au Québec, chaussure imperméable que l’on porte par-dessus une autre chaussure et quine monte guère plus haut que la cheville. Elle se ferme généralement au moyen d'une glissière».

- rubber EN • caoutchouc FR

Fuente: Office québécois de la langue française, 1982

Definición: «Tout couvre-chaussure en toile caoutchoutée ou autre matériau semblable».

En segundo lugar, se procedió a la indagación del término upper y se obtuvo el siguiente resultado:

- upper EN・ tige FR

Fuente: Office québécois de la langue française, 2000: 
Definición: «Partie supérieure de la chaussure excluant le semelage, destinée à habiller et protéger le dessus du pied».

Observaciones: se puede utilizar el término tige o dessus de la chaussure, sin embargo, se desaconseja el término empeigne, concretándose lo siguiente: le terme empeigne est à éviter comme synonyme de tige.

Dicha entrada se presenta junto a la siguiente ilustración:

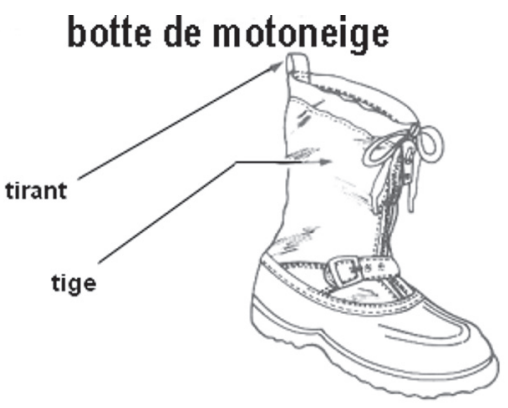

Figura 10: Identificación del término tige como parte de una bota.

Lo anteriormente expuesto nos permite seguir insistiendo en la funcionalidad interpretativa de este tipo de elementos ilustrativos, y de forma análoga, contribuye a esclarecer ambigüedades terminológicas. Es necesario tener en cuenta que la Figura 10 muestra el diseño de una bota y no de un tipo de calzado general, como en los casos anteriormente analizados.

Por último, el estudio de las diferentes opciones aportadas nos conduce a reflexionar sobre la necesidad de actualización del contenido lexicográfico, teniendo en cuenta las fechas de publicación de las fuentes aportadas.

iii. El Diccionario de términos del calzado e industrias afines comprende voces léxicas en español acompañadas de su equivalente y definición en inglés, junto con las correspondientes acepciones y viceversa en inglés. Se realiza, por tanto, la búsqueda del término pala en la sección de español y se obtiene el equivalente en inglés, vamp, junto con la siguiente definición en inglés: «the fore section of the upper,excluding the quarters, which covers the toes and the frontfoot». Esta enunciación incluye el término quarter, cuya búsqueda nos dirige al segundo término objeto de análisis del presente trabajo caña, junto con otras opciones tales como cañeta, cuarto y trasera, y como definición: «pieza de piel que cubre la zona comprendida entre el talón y la pala».

Retomando el análisis del término vamp, se ofrece como sinónimo el término corte (primera acepción), en español y upper, en inglés, coincidiendo con las propuestas encontradas en las fuentes anteriores. La búsqueda de upper en inglés en este mismo recurso nos ofrece como equivalentes: corte, capellada, corte aparado y pala y nos facilita la siguiente definición: «parte superior del calzado que abraza el pie por encima». Entre las acepciones que recoge, cabe señalar upper materials y, en concreto, su definición: «materiales para el corte/la pala; materiales que se emplean para la fabricación de cortes o palas; los más comunes son el cuero, sintéticos y textiles». Se realiza 
también la búsqueda de vamp en inglés, que da como resultados pala, copete y empeine, y como definición: "parte delantera del corte, sin incluir las cañas, que abraza el pie por encima». Como sinónimos aportados en inglés se encuentran upper y full vamp. Por último, el término broguewing se encuentra traducido al español como corte tope y pala, una propuesta que también aparecía recogida en el Diccionario de términos del calzado de la Cámara de Comercio de Alicante.

A grandes rasgos, esta consulta nos llevaría a reconsiderar la necesidad de mejorar la interrelación conceptual entre los términos en este recurso. La posibilidad de la identificación visual de los conceptos se estima como una posible solución que complementaría satisfactoriamente la información léxica y favorecería su identificación conceptual y visual.

b. En lo que respecta al término caña, las posibles traducciones en inglés que se han encontrado son quarter, quarters, leg, legging, quarter topline, top of boogleg, backpart, boot-top, y en francés, únicamente se ha encontrado el término quartier.

i. Empezamos analizando el Diccionario de términos del calzado que nos ofrece, en inglés, varias acepciones para el término caña, como se observa en la Tabla 2, y una única equivalencia en francés.

En primer lugar, nos detenemos a analizar la versión inglesa, en la que observamos que las voces léxicas marcan una distinción entre la caña de una bota y la caña de un zapato, con las equivalencias pertinentes que analizaremos a continuación:

- Para caña (bota) se propone en inglés:

- leg, que tras su búsqueda en el mismo recurso nos da como resultados pierna, pata, caña de la bota. Identificamos también leg of boot que presenta como equivalente en español caña (de la bota).

- legging, que, sin embargo, no aparece como entrada en la sección inglés-español.

- quarter, cuyas equivalencias en español en este diccionario son caña, trasera y cañeta. No encontramos ninguna entrada en la sección sección inglés-español que se identifique con quarter topline.

- Para caña de la bota se propone en inglés top of bootleg y, al realizar la búsqueda del término top en la sección inglés-español, encontramos el término top bootleg como acepción dentro de dicha entrada.

- Para caña (zapato) se propone en inglés quarter. Cabe señalar que el término quarter también se traduce al español como trasera y cañeta.

Se detecta la existencia de diferentes tipos de caña en función de si se trata de un calzado común o una bota, si bien no queda evidenciado con suficiente claridad cómo denominarlo en cada caso en inglés. En la versión inglesa, no aparece ningún tipo de elemento gráfico que haga referencia al término caña, a pesar de que, como se analizó con anterioridad, este recurso lexicográfico incluye ilustraciones en sus anexos.

Una cuestión que nos ha llamado la atención es la presencia de determinados equivalentes en un idioma (por ejemplo, legging o quarter topline propuestos como equivalentes de caña en inglés) que no cuentan con una entrada en la sección del idioma correspondiente. 
Pasamos a analizar la versión bilingüe francés-español, en la que no se identifica ninguna acepción, existiendo un único equivalente denominado quartier. En la versión francesa sí aparece el término quartier identificado gráficamente en el n. 5 de la Ilustración 2 del Anexo n. ${ }^{\circ} 7$, como se observa en la Figura 6; sin embargo, es preciso detenernos a comentar que su equivalente en español en la Figura 6 es tobillera, en lugar de caña, siendo además la única vez que aparece en este diccionario. Cabe enfatizar de igual modo que para la misma parte del calzado (n. ${ }^{\circ}$ ) señalada en la versión francesa como quartier y traducida al español como tobillera, en la versión inglesa se mantiene la traducción al español (tobillera), pero en ingles se traduce como ankle-collar.

Detectamos, por tanto, que se produce nuevamente un caso de incoherencia terminológica que provoca cierta incomprensión y confusión, imposibilitando la correcta interpretación del significado e identificación del equivalente. A pesar de ello, una vez más, el elemento visual contribuye a identificar la parte del calzado a la que se hace referencia y, de esta forma, enfocar la búsqueda en esta dirección.

ii. Le grand dictionnaire terminologique nos ofrece un único equivalente en francés, aunque de nuevo con varias opciones acompañadas de su definición en la misma lengua y su equivalente en inglés. Como se desprende de los resultados recogidos en la Tabla 2, las descripciones aportadas son muy similares y quedan en ciertos casos complementadas por un mismo elemento gráfico que permite al usuario distinguir la parte del calzado en cuestión, de manera que la explicación queda totalmente respaldada, facilitando así su comprensión. A pesar de ello, nos sorprende que no se establezca una diferencia entre la caña de un calzado y la caña de una bota, quedando por tanto de manifiesto cierta carencia semántica en la descripción lexicográfica de término.

iii. En el Diccionario de términos del calzado e industrias afines nos encontramos con tres equivalentes para referirnos a un mismo término. Al igual que ocurría en el Diccionario de términos del calzado, este recurso distingue entre caña, caña de la bota, caña de la pierna y caña del zapato, entre otras acepciones.

- La entrada del término caña, que va acompañada de su definición en inglés: «each of the two back outside parts of a shoe almost symmetrical; they join the vamp at various points according to the design». Como sinónimos, se proponen en español trasera y contrafuerte. Los equivalentes que ofrece en lengua inglesa son quarter, backpart. Se buscaron ambos términos en este mismo diccionario y se obtuvieron los siguientes resultados:

- Quarter: pieza de piel que cubre la zona comprendida entre el talón y la pala. Además, se ofrecen otros términos como equivalentes en español, tales como cañeta, cuarto, trasera. Como sinónimo en inglés, se propone counter.

- Backpart: talonera, parte trasera. Como sinónimo en inglés, se propone quarter.

- La caña de la bota queda definida como: «the part of a boot covering the leg or a portion of it». Como equivalentes en inglés se encuentran las siguientes propuestas: leg of the boot, boot-top, boot-leg. Como sinónimos, se proponen en español frontal de la bota y bota de caña alta.

- Para la caña de la pierna se proponen dos definiciones:

a) top of the bootleg;

b) the perimeter of the leg just under the knee. 
- La caña del zapato se define como: «the edge of the upper round the throat of the shoe or boot». Se propone como equivalente al inglés top line y como sinónimo en español cinta de refuerzo de la caña.

La valoración es similar al caso anterior, en tanto que las diferentes propuestas terminológicas aportadas para un mismo concepto precisarían de una cohesión interna más precisa que facilite al usuario interpretación, así como de un matiz pragmático que resulta de utilidad al traductor para poder decidir en cada caso el equivalente exacto o bien tener la seguridad de que puede utilizar indistintamente un término y otro, como sinónimos, sin que exista un abanico de posibilidades tan amplio que lo que provoca es una mayor indecisión. Se pone claramente de manifiesto y reiteramos en que la presencia de elementos multimodales aportaría un gran valor lexicográfico a dicho recurso, pudiendo incluso salvar las inconveniencias antes referidas.

A modo de resumen, se ha elaborado la siguiente tabla que recoge los problemas e inconvenientes a nivel lexicográfico que se han detectado a lo largo de nuestro análisis en las obras de consulta objeto de estudio.

\begin{tabular}{|c|c|c|c|}
\hline Problemas relacionados con: & $\begin{array}{l}\text { Diccionario de } \\
\text { términos del calzado }\end{array}$ & $\begin{array}{l}\text { Le grand dictionnaire } \\
\text { terminologique }\end{array}$ & $\begin{array}{l}\text { Diccionario de } \\
\text { términos del calzado } \\
\text { e industrias afines }\end{array}$ \\
\hline Existencia de un equivalente unívoco & + & $\begin{array}{l}\text { En el caso del } \\
\text { término «pala» }\end{array}$ & - \\
\hline Coherencia terminológica & + & + & - \\
\hline $\begin{array}{l}\text { Interrelación conceptual } \\
\text { entre términos }\end{array}$ & + & + & + \\
\hline Definición del término poco precisa & - & + & - \\
\hline $\begin{array}{l}\text { Uso de términos especializados } \\
\text { que dificultan la comprensión de la } \\
\text { definición }\end{array}$ & - & + & + \\
\hline $\begin{array}{l}\text { Cohesión entre las unidades de } \\
\text { significación verbal y no verbal }\end{array}$ & + & + & - \\
\hline Entradas o voces no actualizadas & & + & - \\
\hline $\begin{array}{l}\text { Ausencia de elementos multimodales } \\
\text { que resultarían necesarios para } \\
\text { facilitar la comprensión }\end{array}$ & - & - & + \\
\hline
\end{tabular}

Tabla 3. Identificación de los problemas encontrados durante la consulta de los términos pala y caña en las fuentes lexicográficas consultadas. 


\section{Conclusión}

Basándonos en los resultados obtenidos, queda de manifiesto el carácter lexicográfico restrictivo de las fuentes objeto de estudio, no solo por la limitación a descripciones semánticas y propuestas de equivalentes terminológicos en los idiomas correspondientes o la evidencia de cierta incoherencia terminológica respecto a las equivalencias propuestas, sino también por la incorrecta funcionalidad y referencia a los elementos multimodales.

Las relaciones intersemióticas entre las imágenes y el texto aportan coherencia y cohesión a la interpretación del contenido semántico que ofrecen este tipo de fuentes. Como hemos visto, las formas de representación lingüísticas y no lingüísticas, si actúan como un todo (Martinec y Salway 2005) coherente, correcto y lógico, proporcionan información contextual (Gómez González-Jover y Vargas Sierra, 2004, s.p.) que facilita la identificación terminológica; su ausencia la dificulta y su uso incorrecto genera incomprensión, provocando la no satisfacción del fin último de un diccionario y llevando al traductor a cierta inseguridad a la hora de decidirse por la elección de un término $\mathrm{u}$ otro en el idioma de destino.

En ciertos casos, se desconoce la posibilidad de que se pueda utilizar indistintamente un término u otro, es decir, si las diferentes propuestas encontradas se deben a una sinonimia exacta o no. Del mismo modo, se ignora y no existe referencia al contorno ${ }^{8}$ lexicográfico, es decir, según el Diccionario de la lengua española: «en lexicografía, conjunto de los elementos de la definición que informan sobre el contexto habitual del vocablo definido, en oposición a los elementos que informan sobre su contenido».

Dicha experiencia práctica nos ha demostrado que la recurrencia de forma exclusiva a diccionarios especializados no es suficiente para satisfacer el objetivo de la labor traductora. Este tipo de materiales están concebidos, en muchas ocasiones, todavía como repertorios o glosarios bilingües, que se alejan de la concepción actual del diccionario, de las nuevas necesidades comunicativas y resultan insuficientes para satisfacer las necesidades del traductor especializado. Éste se ve obligado hoy en día a recolectar información de diferentes fuentes, consultar a expertos en la materia, y al mismo tiempo trabajar a contrarreloj frente a los límites temporales impuestos por la actividad profesional.

Este análisis contrastivo nos muestra que las herramientas de consulta lexicográfica no están diseñadas exclusivamente para cubrir las necesidades traductológicas, ni tampoco actualizadas a las necesidades comunicativas actuales, en las que impera el uso preferente de recursos multimodales y digitales que apelen a los sentidos de los usuarios. Los recursos multimodales ofrecen un amplio abanico de posibilidades que contemplan la recurrencia a ilustraciones, fotografías o imágenes reales, imágenes en movimiento o GIF, vídeos explicativos que complementen la definición o incluso la reemplacen, audios, entre otros. Lamentablemente, queda mucho camino por recorrer hasta que el traductor tenga acceso a herramientas lexicográficas que integren este tipo de elementos que tanto podrían beneficiar a la labor traductora.

Una cuestión que se plantea como propuesta para un estudio futuro sería la elaboración de un diccionario adaptado a las innovaciones tecnológicas actuales, que, además de ofrecer un acceso rápido y eficiente a la información, pusiera a disposición del traductor información lingüística 
y no lingüística, que complementara la representación conceptual y la exactitud de la misma, contribuyendo a la interpretación de su significado y la posterior producción en la lengua meta.

\section{Referencias bibliográficas}

Abaitua, J. (2001). Memorias de traducción en TMX compartidas por Internet. Tradumàtica: traducció i tecnologies de la informació i la comunicació. Universitat Autònoma de Barcelona.

Alcaraz-Varó, E. et al. (2006). Diccionario De Términos Del Calzado e Industrias Afines: inglés-español, Spanish-English. Barcelona: Ariel.

Cabré, M. T. (2010). La Terminología: representación y comunicación: Elementos para una teoría de base comunicativa y otros artículos. Documenta Universitaria.

- (2005). Hacia una teoría comunicativa de la terminología: aspectos metodológicos. Hacia una teoría comunicativa de la terminología: aspectos metodológicos, 1000-1022.

- (1993) La terminología: Teoría, metodología, aplicaciones. Barcelona: Antártida/Empúries.

Cabré, M.; Feliu, J.; \& Tebé, C. (2001). Bases cognitivas de la terminología: hacia una visión comunicativa del concepto. Sendebar, 12, 301-310.

Cámara de Comercio de Alicante (2001). Diccionario de términos del calzado inglés-español, español-inglés. https://www.manuelsamper.es/utilidades/utilidad_3.pdf

Cámara de Comercio de Alicante (2001). Diccionario de términos del calzado francés-español, español-francés. https://www.manuelsamper.es/utilidades/utilidad_4.pdf

Cárdenas, B. S. (2008). De los diccionarios para traducir o lo que no necesita el traductor. In 25 años de lingüística en España [Recurso electrónico]: hitos y retos= 25 years of applied linguistics in Spain: milestones and challenges (pp. 1101-1113). Universidad de Murcia.

Faber, P.; León Araúz, P.; Prieto-Velasco, J. A.; \& Reimerink, A. (2007). Linking Images and Words: the description of specialized concepts. International Journal of Lexicography, 20 (1), 39-65.

Gamero Pérez, S. (2001). La traducción de textos técnicos: descripción y análisis de textos (alemán-español). Barcelona: Ariel.

García Ubago, M. M. (2012). Traducción del lenguaje de especialidad [Apuntes de terminología del grado de traducción e interpretación]. Soria: Universidad de Valladolid.

Gómez de Enterría, J. (1998). Aproximación a los textos científicos. Una metodología activa. Comunicación presentada en el XVI Congreso Nacional de Lingüística Aplicada, Logroño - AESLA.

Gómez González-Jover, A.; \& Vargas Sierra, C. (2004). Aspectos metodológicos para la elaboración de diccionarios especializados bilingües destinados al traductor. In El español, lengua de traducción. II congreso internacional (pp. 365-398). Bruselas: ESLEtRA.

Kress, G.; \& van Leeuwen, T. (2001). Multimodal Discourse: The modes and Media of Contemporary Communication. London: Arnold.

Le grand dictionnaire terminologique http://www.granddictionnaire.com/

Martinec, R.; \& Salway, A. (2005). A system for image-text relations in new (and old) media. Visual Communication, 4, 337-371.

Porto Dapena, A. (2002). Manual de técnica lexicográfica. Madrid: Arco/Libros. 
Prieto Velasco, J. A.; \& Faber, P. (2012). Graphical information. In A cognitive linguistics view of terminology and specialized language (pp. 225-248) .Berlin, Boston: De Gruyter Mouton.

Sánchez Cárdenas, B. (2008). De los diccionarios para traducir o lo que no necesita el traductor. In 25 años de lingüística en España [Recurso electrónico]: hitos y retos= 25 years os applied linguistics in Spain: milestones and challenges (pp. 1101-1113). Universidad de Murcia. 